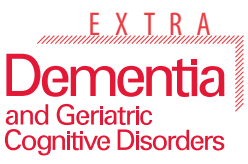

\begin{tabular}{l|l}
\hline Dement Geriatr Cogn Disord Extra 2014;4:335-343 \\
\hline DOI: 10.1159/000363500 & $\begin{array}{l}\text { c 2014 S. Karger AG, Basel } \\
\text { 1664-5464/14/0042-0335 } \$ 39.50 / 0 \\
\text { wwww.karger.com/dee }\end{array}$ \\
& \\
\hline
\end{tabular}

This is an Open Access article licensed under the terms of the Creative Commons AttributionNonCommercial 3.0 Unported license (CC BY-NC) (www.karger.com/OA-license), applicable to the online version of the article only. Distribution permitted for non-commercial purposes only.

\title{
Agitation in Dementia: Relation to Core Cerebrospinal Fluid Biomarker Levels
}

\author{
Victor Bloniecki ${ }^{a}$ Dag Aarsland ${ }^{b, c}$ Jeffrey Cummings ${ }^{\text {e Kaj Blennow }}{ }^{c}$ \\ Yvonne Freund-Levi ${ }^{a}$ \\ a Division of Clinical Geriatrics, b Center for Alzheimer Research, Division for Neurogeriatrics, \\ Department of Neurobiology, Caring Sciences and Society (NVS), Karolinska Institutet, \\ Karolinska University Hospital Huddinge, Stockholm, and ${ }^{\complement} C$ Clinical Neurochemistry \\ Laboratory, Department of Psychiatry and Neurochemistry, Institute of Neuroscience and \\ Physiology, Sahlgrenska University Hospital, The Sahlgrenska Academy at University of \\ Gothenburg, Mölndal, Sweden; ${ }^{c}$ Center for Age-Related Medicine, Stavanger University \\ Hospital, Stavanger, Norway; ${ }^{~}$ Cleveland Clinic Lou Ruvo Center for Brain Health, Las Vegas, \\ Nev., USA
}

\section{Key Words}

Dementia · Agitation · CMAI · Cerebrospinal fluid (CSF) · Dementia biomarkers ·

Neurofibrillary tangles · Behavioral and psychological symptoms in dementia (BPSD)

\section{Abstract}

Background: The objective of this study was to examine the associations of agitation with the cerebrospinal fluid dementia biomarkers total-tau (T-tau), phosphorylated-tau (P-tau) and $A \beta_{1-42}$. Methods: One hundred patients (mean age $\pm S D, 78.6 \pm 7.5$ years) with dementia and neuropsychiatric symptoms, of whom $67 \%$ were female, were included. Agitation was measured using the Cohen-Mansfield Agitation Inventory (CMAI; $46.5 \pm 11.8$ points). Results: Total CMAI correlated with T-tau $\left[r_{\mathrm{s}}(31)=0.36, \mathrm{p}=0.04\right]$ and P-tau $\left[r_{\mathrm{s}}(31)=0.35, \mathrm{p}=0.05\right]$ in patients with Alzheimer's disease (AD; $n=33$ ) but not in the total dementia population $(n=$ 95). Conclusions: Our results suggest that tau-mediated pathology including neurofibrillary tangles and the intensity of the disease process might be associated with agitation in AD.

(C) 2014 S. Karger AG, Basel

V.B. and Y.F.-L. share the first authorship. Trial registration: Karolinska University Hospital identifier study ID: 20100039. 


\section{Background}

The global prevalence of dementia is increasing and it is predicted to affect over 80 million individuals worldwide by the year of 2040 [1]. The dementia syndrome includes a progressive deterioration of cognitive functions often accompanied by neuropsychiatric symptoms referred to as behavioral and psychological symptoms in dementia (BPSD) $[2,3]$.

BPSD can be divided into three main clusters: agitation, psychosis and mood disorders [4]. Agitation includes both emotional distress and behavioral changes such as aggression, irritability, pacing and restlessness [4]. Agitation is experienced by approximately $20 \%$ of the dementia patients in a community setting and increases up to $50 \%$ in institutionalized patients [5]. Overall, more than $90 \%$ of the patients with dementia suffer from at least one BPSD symptom during the course of the disease [6]. BPSD are major contributors to increased disease burden for both patients and caregivers, reduce the ability to perform activities of daily living, contribute to earlier hospitalization and constitute approximately $30 \%$ of the dementia-associated treatment costs [7-10].

Overall, the research regarding the pathology of BPSD has been limited. Animal models using male transgenic APP/PS1 mice resulting in rapid progression of amyloid neuropathology have been shown to exhibit behavioral symptoms including disinhibition such as impulsivity, reduced body weight and enhanced aggression relative to wild-type controls [11]. Agitation has also been associated with the presence of neurofibrillary tangles (NFT). In a postmortem study of patients with severe Alzheimer's disease (AD), increased cerebral burden of NFTs in the orbitofrontal cortex was found to correlate with agitation [measured by the Neuropsychiatric Inventory (NPI) agitation subscale] [12]. In a study from 2002, an aggressive behavior cluster including actions such as physical aggression, aggressive resistance and verbal aggression was associated with loss of neurons in the rostral locus coeruleus [13]. Furthermore, it is very possible that abnormalities in cerebral neurotransmission are involved in the cause of BPSD [14].

Recent studies have shown that the cerebrospinal fluid (CSF) biomarkers totaltau (T-tau), phosphorylated-tau (P-tau) and the 42 amino acid isoform of $\beta$-amyloid protein $\left(A \beta_{1-42}\right)$ can identify AD with high specificity and sensitivity [15-17]. Much data suggest that the CSF level of T-tau reflects the intensity of the neuronal degeneration, while P-tau levels correlate with cortical NFT load [for review, see 15]. In contrast, less is known about the relationship between these biomarkers and BPSD. In a recent study, apathy was shown to correlate with increased levels of both P-tau and T-tau in CSF, suggesting that apathy could be associated with increased amounts of NFTs in the brain [18]. The association between depression and AD biomarkers has also been studied, but no evidence that links depression with the presence of amyloid plaques or NFTs was found $[18,19]$. Levels of CSF A $\beta_{1-42}$ have been shown to display a negative correlation with increased aggressive behavior in demented patients [20]. Several studies indicate that NFTs and amyloid plaques could be involved in the pathological process causing BPSD but this has not been proven and further research is warranted $[11,12,18-20]$. Current pharmacological treatment options for agitation mainly include antipsychotics, which display modest efficacy and might be associated with severe cerebrovascular events [21,22]. This accentuates the importance of identifying potential targets for novel drug development.

The primary aim of this study was to examine the association between agitation as measured by the Cohen-Mansfield Agitation Inventory (CMAI) and the CSF biomarkers T-tau, P-tau and $A_{\beta 1-42}$ in patients with dementia and BPSD. 


\section{Materials and Methods}

\section{Patients}

One hundred and forty-five patients living in the community were referred from general practitioners to the Memory Clinic of the Department of Geriatric Medicine at Karolinska University between January 2003 and September 2005. One hundred patients who fulfilled the criteria according to the Diagnostic and Statistical Manual of Mental Disorders, fourth edition (DSM-IV) [23] for the diagnosis of Alzheimer's dementia with behavioral disturbance, vascular dementia with behavioral disturbance and mixed dementia with behavioral disturbance were included in the study. A total score of at least 10 on the NPI [24] with symptoms for at least 2 weeks prior to inclusion was required. Patients were excluded if they had a diagnosis of schizophrenia or other psychiatric disorders according to the DSM-IV, a history of seizures, active peptic ulcer or clinically significant hepatic, renal, pulmonary or metabolic disturbances. None of the patients were receiving any kind of dementia medication such as cholinesterase inhibitors or neuroleptics prior to inclusion, although some patients were medicated with psychotropics $(n=48)$ including antidepressants, benzodiazepines and antihistamines at the time of study entry. The included patient data is part of a larger study that is currently in preparation, but for further information regarding the study population, assessment and other study specifics, please see the original article [25].

\section{Clinical Assessment}

Diagnostic procedures included somatic, psychiatric, neurological and neuroimaging (CT scans) examination performed by a licensed specialist in geriatric medicine with experience in dementia (Y.F.-L.). In addition to a clinical interview of patients and caregivers, a standardized scale of cognition [Mini-Mental State Examination (MMSE)] [26] and neuropsychiatric symptoms (NPI) was administered for diagnostic purposes. Lumbar punctures were performed and CSF was collected according to the standard operation procedures for dementia investigation existing in Sweden, in which lumbar punctures are a part of the clinical approach to dementia diagnosis. Lumbar puncture was successfully performed in 95 of the 100 included patients ( 3 patients refused the procedure, 1 patient had an arachnoid cyst and in 1 patient, LP was unsuccessful due to muscular rigidity.)

The level of agitation was assessed using the CMAI [27], which consists of 29 subitems related to agitation that are measured and rated based on frequency 1-7 ( $1=$ never, $7=$ a couple of times every hour). The total CMAI is calculated as the sum of the frequency scores with a maximum score of 203. The CMAI can be separated into four subitems relating to different subtypes of agitation: subitem 1 = aggressive physical behavior such as kicking or hitting, subitem 2 = nonaggressive physical behavior such as pacing, subitem $3=$ verbal aggressive behavior such asscreaming and subitem 4 = verbal nonaggressive behavior including negativism or complaining.

Of the 95 patients who underwent a successful lumbar puncture, 35\% were diagnosed with $\mathrm{AD}, 27 \%$ had mixed $\mathrm{AD}$ with vascular factors, $18 \%$ had vascular dementia, $12 \%$ were diagnosed with mild cognitive impairment and $8 \%$ were diagnosed with unspecified dementia. Sixty-three (66\%) were females, the mean age was 78.6 years $(\mathrm{SD} \pm 7.5)$ and the mean total CMAI score was 46.3 (SD \pm 11.8 ).

\section{CSF Analyses}

CSF ( $6 \mathrm{ml}$ ) was collected and then stored in polypropylene tubes. The first $2 \mathrm{ml}$ of every sampling was discarded and the rest was centrifuged at 3,000 rpm for $10 \mathrm{~min}$ at $+4^{\circ} \mathrm{C}$ and frozen in aliquots of $2 \mathrm{ml}$ at $-70^{\circ} \mathrm{C}$. The collected CSF was used for analysis of T-tau, P-tau and $\mathrm{A} \beta_{1-42}$ at the Department of Clinical Neurochemistry, Mölndal Hospital. The T-tau concentration in CSF was determined using a sandwich ELISA (INNOTEST ${ }^{\circledR}$ hTAU Ag; Innogenetics, Gent, 
Bloniecki et al.: Agitation in Dementia: Relation to Core Cerebrospinal Fluid Biomarker Levels

Table 1. Baseline characteristics of the included patients

\begin{tabular}{|c|c|c|}
\hline Variables & All $(n=95)$ & $\mathrm{AD}(\mathrm{n}=33)$ \\
\hline Age, years & $78.6 \pm 7.5$ & $78.7 \pm 7.6$ \\
\hline Females, $\mathrm{n}$ & 63 & 19 \\
\hline MMSE (0-30 points) & $20.2 \pm 4.6$ & $19.1 \pm 4.2$ \\
\hline Total CMAI (0-203 points) & $46.3 \pm 11.8$ & $44.6 \pm 10.7$ \\
\hline CMAI aggressive physical behavior ( 0 - 77 points) & $11.6 \pm 2.0$ & $11.6 \pm 2.6$ \\
\hline CMAI nonaggressive physical behavior ( $0-70$ points) & $17.3 \pm 6.6$ & $17.3 \pm 6.1$ \\
\hline CMAI verbal aggressive behavior ( $0-21$ points) & $4.9 \pm 2.3$ & $4.5 \pm 1.9$ \\
\hline CMAI verbal nonaggressive behavior ( $0-35$ points) & $12.5 \pm 5.7$ & $11.2 \pm 5.3$ \\
\hline T-tau, pg/ml & $775.4 \pm 527.6$ & $767.6 \pm 472.9$ \\
\hline P-tau, pg/ml & $97.4 \pm 58.1$ & $94.8 \pm 44.9$ \\
\hline $\mathrm{A} \beta_{1-42}, \mathrm{pg} / \mathrm{ml}$ & $478.2 \pm 182.4$ & $464.4 \pm 141.0$ \\
\hline
\end{tabular}

All values are expressed as means $\pm \mathrm{SD}$, except for the number of females.

Belgium) specifically constructed to measure all tau isoforms irrespectively of phosphorylation status [28]. Tau phosphorylated at threonine 181 (P-tau181) was measured using a sandwich ELISA method [INNOTEST ${ }^{\circledR}$ PHOSPHO-TAU (181P); Innogenetics] [29]. A $\beta_{1-42}$ levels were determined using a sandwich ELISA method [INNOTEST ${ }^{\circledR} \beta$-AMYLOID (1-42); Innogenetics] specifically constructed to measure $A \beta$ containing both the first and the $42 \mathrm{nd}$ amino acid [30]. In this assay, the monoclonal antibody $21 \mathrm{~F} 12$, which is highly specific to the C-terminus of $\mathrm{A} \beta_{1-42}$, was used for capture, and 3D6, which is specific to the N-terminus, was used as detector.

\section{Statistics}

Since the investigated variables are scores from ordinal scales, it can be argued that nonparametrical methods are better suited than parametrical ones. For associations between the included variables, Spearman's rank order correlation was used. To adjust for potential confounders, all significant results were adjusted for age, gender and the level of cognitive impairment (MMSE) using linear regression, and all results where validated for normality, linearity and equal variance. The sample size was relatively small so that nonparametric regression could not be used since it requires larger sample sizes than regression based on parametric methods. Data are given as means and 95\% confidence intervals (CI) or as stated, and $\mathrm{p}$ values $<0.05$ were considered statistically significant. All statistical analyses were carried out with the Statistica ${ }^{\circledR} 10.0$ software package (StatSoft, Tulsa, Okla., USA).

\section{Ethics}

Informed consent was obtained from the subjects or their legally acceptable representative prior to inclusion. The regional Ethics Committee of Karolinska Institute, Stockholm, Sweden, approved the study (registration number 441/01 at Karolinska Institutet).

\section{Results}

Characteristics of the cohort are shown in table 1.

Relation between Agitation and AD Biomarkers in CSF

No statistically significant correlations were found between total CMAI, CMAI subscores and the CSF biomarkers T-tau, P-tau and $A \beta_{1-42}$ when analyzing all included patients $(n=95)$. 


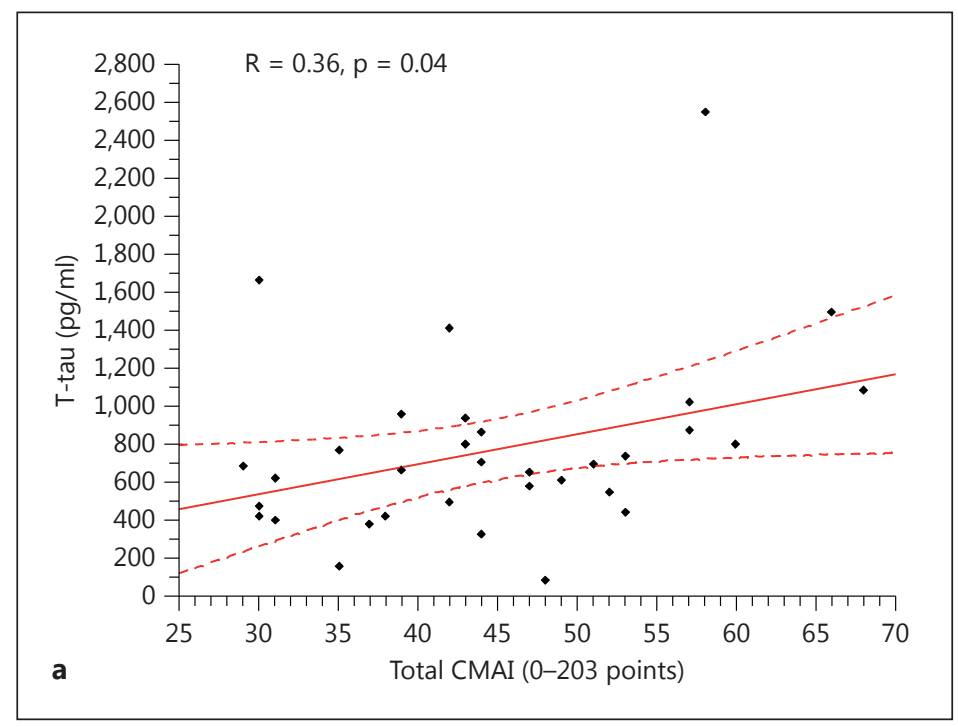

Fig. 1. Scatterplots of Spearman's correlations between dementia biomarkers in CSF and total CMAI. a Scatterplot of the correlation between T-tau and total CMAI in AD patients. b Scatterplot of the correlation between P-tau and total CMAI in AD patients. c Scatterplot of the correlation between Ptau and total CMAI in all patients.
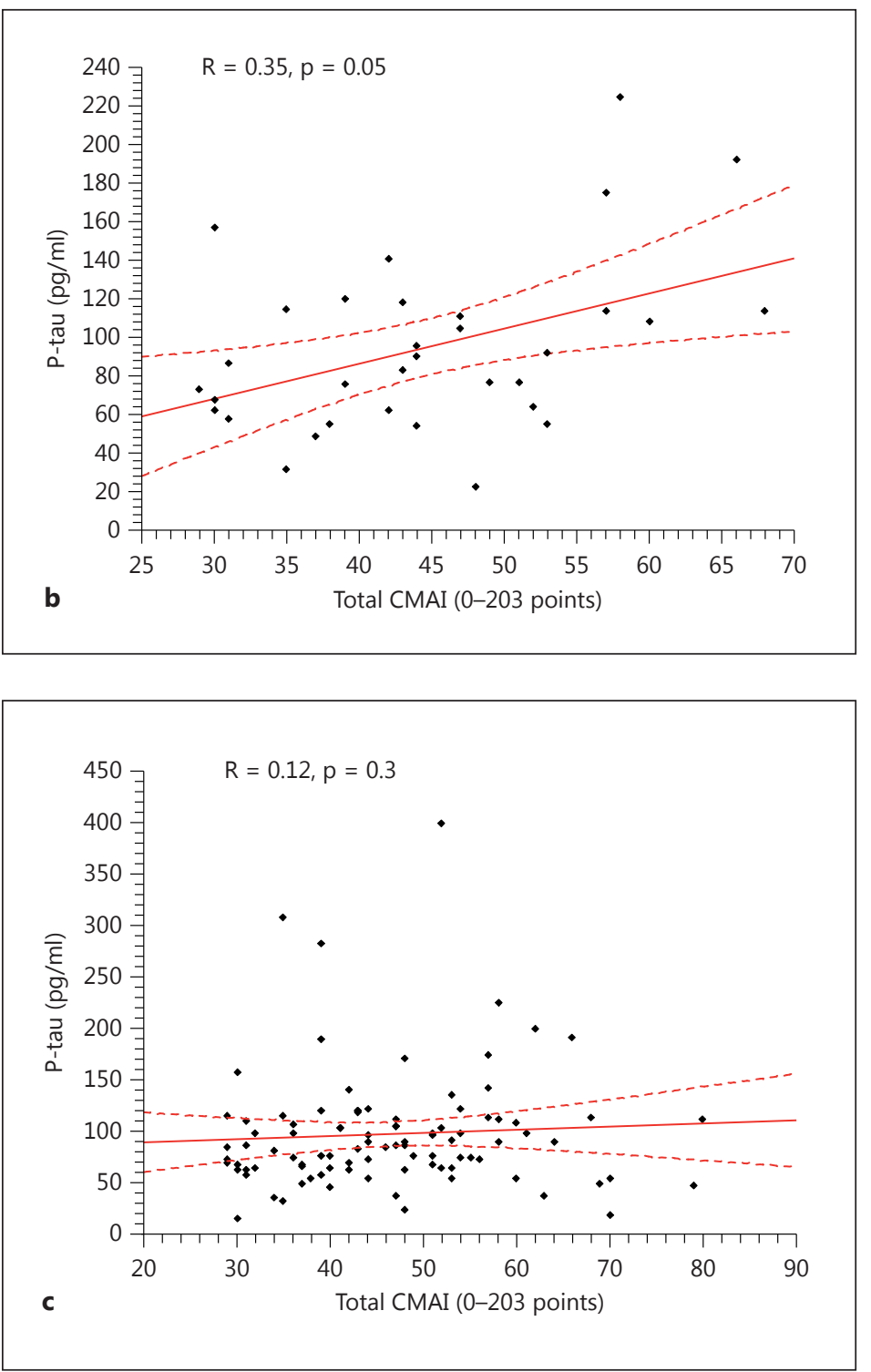


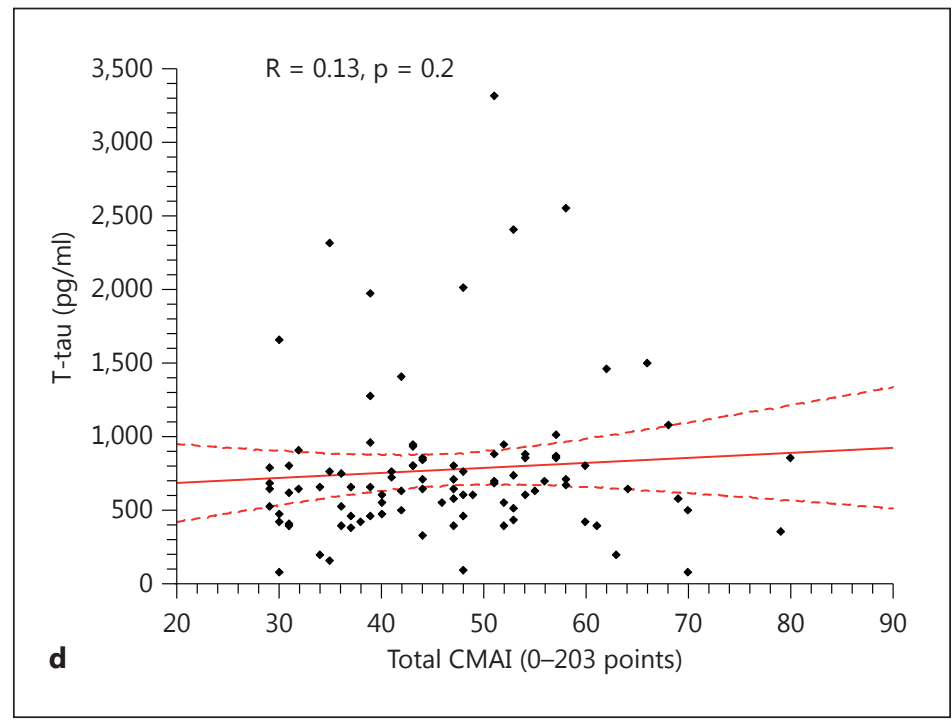

Fig. 1. Scatterplots of Spearman's

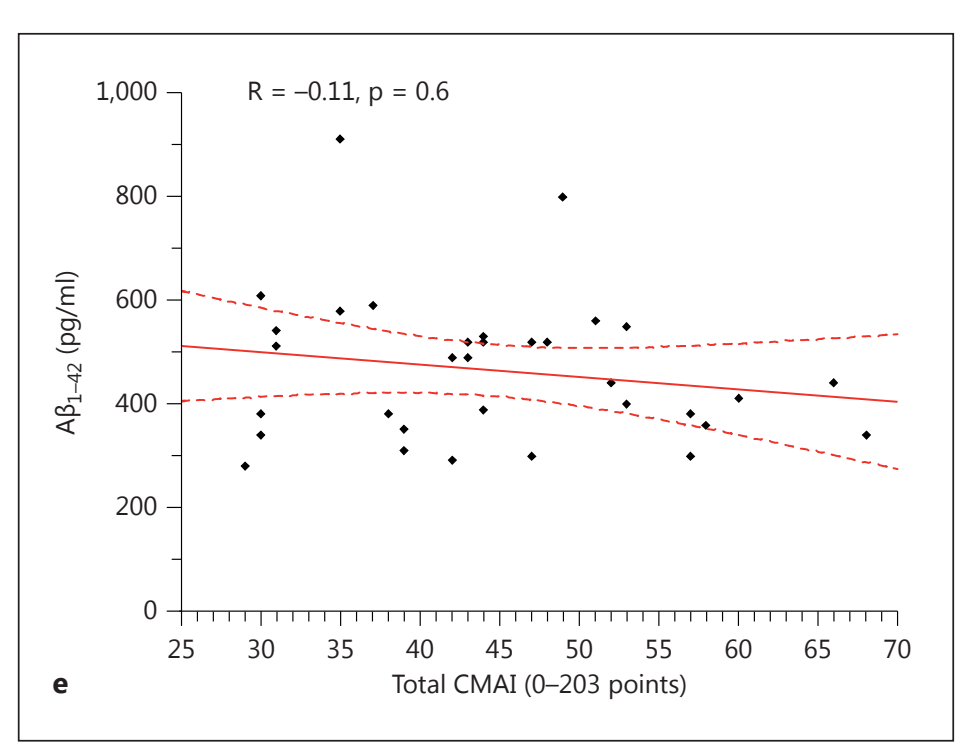
biomarkers in CSF and total CMAI. d Scatterplot of the correlation between T-tau and total CMAI in all patients. e Scatterplot of the correlation between $A \beta_{1-42}$ and total CMAI in $\mathrm{AD}$ patients.

Within the AD group $(n=33)$ both T-tau $\left[r_{s}(31)=0.36, p=0.04\right]$ and P-tau $\left[r_{s}(31)=0.35\right.$, $\mathrm{p}=0.05$ ] (Spearman's rank correlation) were significantly correlated with total CMAI (fig. 1). AD patients also displayed a significant correlation between T-tau and CMAI subitem nonaggressive physical behavior $\left[r_{s}(31)=0.4, p=0.02\right]$. There were no significant associations between the levels of $A \beta_{1-42}$ and agitation (total CMAI) $\left[r_{s}(31)=-0.11, p=0.6\right]$ in the AD patients. No other significant correlations were found in the AD group. No significant correlations were found between any of the dementia biomarkers and total CMAI in the other dementia subgroups (data not shown). Correlation analyses are shown in table 2.

Linear regression analysis confirmed the correlations between agitation and the AD biomarkers in the AD subgroup. When adjusted for age, gender and MMSE score, T-tau displayed a significant correlation with total CMAI $[\mathrm{F}(1,28) 4.45, \mathrm{p}=0.04]$. The analysis showed that a one-unit increase of T-tau results in a 0.008-unit increase in total CMAI. The association between P-tau and total CMAI remained significant after adjustment for all aforementioned variables $[F(1,28) 7.16, p=0.01]$. A one-unit increase of P-tau was associated with 
Table 2. Correlations between agitation and dementia biomarkers in CSF

\begin{tabular}{lcc}
\hline Variables & All $(\mathrm{n}=95)$ & AD $(\mathrm{n}=33)$ \\
\hline Total CMAI and T-tau & $0.13(\mathrm{p}=0.2)$ & $0.36(\mathrm{p}=0.04)$ \\
Total CMAI and $\mathrm{A} \beta_{1-42}$ & $0.04(\mathrm{p}=0.7)$ & $-0.11(\mathrm{p}=0.6)$ \\
Total CMAI and P-tau & $0.12(\mathrm{p}=0.3)$ & $0.35(\mathrm{p}=0.05)$
\end{tabular}

Spearman's correlation was used for statistical calculations.

a 0.11-unit increase in the total CMAI score. The correlation between T-tau and nonaggressive physical behavior remained significant after adjustment for confounders, and a one-unit increase of P-tau was associated with a 0.05 -unit increase in the CMAI subitem score.

\section{Discussion}

This is one of the first studies analyzing levels of CSF AD biomarkers in patients with significant neuropsychiatric symptoms and dementia. The main finding was a significant relationship between agitation and CSF levels of both T-tau and P-tau in patients with AD. Based on our previous research [31], these results suggest that the cerebral NFT burden is associated with agitation in dementia. These findings are also consistent with previous research showing a correlation between agitation and NFT burden in AD patients post mortem [12]. T-tau was also correlated with agitation, suggesting that agitation is associated with the amount of axonal degeneration and the intensity of the disease process [31]. Furthermore, our results are supported by the fact that they remained significant even after performing multivariable linear regression to adjust for the effect of dementia severity as measured by MMSE, since it is known that the dementia severity is associated with agitation. The taumediated pathology is unlikely to be a direct cause of agitation in AD but rather a part of a complicated network of different pathological mechanisms. The neuronal damage caused by the NFTs could affect neurotransmission resulting in agitation, as has previously been proposed [14], and a study including both neurotransmitters and biomarkers would facilitate further investigation of these associations. Previous research has indicated that frontal lobe circuit dysfunction, possibly involving tau-mediated pathology, could be a cause of agitation in dementia [32]. Although our results are significant, a previous study comparing agitation in AD patients with CSF-biomarkers contradicts these findings [18]. Possible explanations for this discrepancy are the facts that the NPI subitem score for agitation was used to quantify agitation in that study, which is less sensitive as compared to CMAI, and that the study only included patients with mild AD not selected for BPSD.

When all dementia subjects were included, there were no significant correlations between agitation and CSF biomarkers. This suggests that the association of agitation with the burden of NFTs is restricted to AD patients only and not valid in the general dementia population. No significant correlation was found between agitation and $A \beta_{1-42}$ in the $A D$ group. Other studies have also failed to find an association between agitation and $A \beta_{1-42}$ [18]. Although our result contradicts findings from a previous report that found a negative correlation between aggressive behavior and $A \beta_{1-42}$ [20], the current study did not include adjustment for severity of dementia, which might have contributed to this association. Since amyloid plaques are such an integral phenomenon of the pathophysiological process of $\mathrm{AD}$, it is reasonable to believe that they are at least partially involved in the mechanism causing agitation. A possible explanation for the lack of association between agitation and $A \beta_{1-42}$ could be that amyloid deposi- 
tions are an upstream process to agitation, eventually leading to the presence of BPSD symptoms through mechanisms such as neuronal dysfunction or altered neurotransmission. This theory is supported by the recent study finding that abnormal levels of AD biomarkers, including $A \beta_{1-42}$ and displaying a pattern of preclinical $\mathrm{AD}$ in healthy individuals, predicts an increased presence of BPSD in the future [33].

Several limitations of this study have to be taken into account. BPSD tend to fluctuate over time [34]. The association between agitation and dementia biomarkers may therefore change over time, and thus longitudinal studies may better capture this association. Some patients were also treated with stable psychotropic medications, and this may have influenced our findings. In addition, the participants were selected from referrals to a university hospital and might thus have been biased.

In conclusion, our study provides added understanding of the pathological mechanism of BPSD in patients with dementia and suggests that tau-mediated pathology is associated with agitation in AD. Further exploration of the possible pathological mechanism mediated by NFT is of interest in order to pinpoint and clarify the exact pathology associated with BPSD and to provide a basis for the development of patient-tailored pharmacological and therapeutic intervention.

\section{Acknowledgments}

We want to express our gratitude to the patients, caregivers and staff at the Outpatient Memory Clinic and Inpatient Wards at the Department of Geriatrics, Karolinska University Hospital Huddinge, Stockholm, Sweden.

\section{Disclosure Statement}

The present study is an academically initiated study initially financed by an independent grant from Janssen Pharmaceuticals, Stockholm, Sweden. The company was represented in the trial steering committee with regard to the study design but was not involved in patient collection or analyses of scientific data. This work was also supported by grants from the Petrus and Augusta Hedlunds Foundation as well as the Demensförbundet and Loo and Hans Osterman Foundation for Medical Research, Karolinska Institutet. J.C. owns the copyright of the NPI.

\section{References}

1 Ferri CP, Prince M, Brayne C, Brodaty H, Fratiglioni L, Gagnuli M, et al: Global prevalence of dementia: a Delphi consensus study. Lancet 2005;366:2112-2117.

2 Ritchie K, Lovestone S: The dementias. Lancet 2002;360:1759-1766.

-3 McKeith I, Cummings J: Behavioural changes and psychological symptoms in dementia disorders. Lancet Neurology 2005;4:735-742.

4 Ballard C, Day S, Sharp S, Wing G, Sorensen S: Neuropsychiatric symptoms in dementia: importance and treatment considerations. Int Rev Psychiatry 2008;20:396-404.

5 Lyketsos CG, Lopez O, Jones B, Fitzpatrick AL, Breitner J, DeKosky S: Prevalence of neuropsychiatric symptoms in dementia and mild cognitive impairment: results from the cardiovascular health study. JAMA 2002;288: 1475-1483.

6 Ballard C, Corbett A: Management of neuropsychiatric symptoms in people with dementia. CNS Drugs 2010; 24:729-739.

7 Grossberg GT: The ABC of Alzheimer's disease: behavioral symptoms and their treatment. Int Psychogeriatr 2002;14:27-49. 
Bloniecki et al.: Agitation in Dementia: Relation to Core Cerebrospinal Fluid Biomarker Levels

-8 Schnaider Beeri M, Werner P, Davidson M, Noy S: The cost of behavioral and psychological symptoms of dementia (BPSD) in community dwelling Alzheimer's disease patients. Int J Geriatr Psychiatry 2002;17:403408.

-9 Cerejeira J, Lagarto L, Mukaetova-Ladinska EB: Behavioral and psychological symptoms of dementia. Front Neurol 2012;3:73.

-10 Allegri RF, Sarasola D, Serrano CM, Taragano FE, Arizaga RL, Butman J, et al: Neuropsychiatric symptoms as a predictor of caregiver burden in Alzheimer's disease. Neuropsychiatr Dis Treat 2006;2:105-110.

11 Pugh PL, Richardson JC, Bate ST, Upton N, Sunter D: Non-cognitive behaviours in an APP/PS1 transgenic model of Alzheimer's disease. Behav Brain Res 2007;178:18-28.

12 Tekin S, Mega MS, Masterman DM, Chow T, Garakian J, Vinter HV, et al: Orbitofrontal and anterior cingulate cortex neurofibrillary tangle burden is associated with agitation in Alzheimer disease. Ann Neurol 2001;49: 355-361.

13 Matthews KL, Chen CPL, Esiri MM, Keene J, Minger SL, Francis PT: Noradrenergic changes, aggressive behavior, and cognition in patients with dementia. Biol Psychiatry 2002;51:407-416.

14 Lanari A, Amenta F, Silvestrelli G, Tomassoni D, Pernetti L: Neurotransmitter deficits in behavioural and psychological symptoms of Alzheimer's disease. Mech Ageing Dev 2006;127:158-165.

$\checkmark 15$ Blennow K, Hampel H, Weiner M, Zetterberg H: Cerebrospinal fluid and plasma biomarkers in Alzheimer's Disease. Nat Rev Neurol 2010;6:131-144.

-16 Prvulovic D, Hampel H: Amyloid $\beta(\mathrm{A} \beta$ ) and phospho-tau (p-tau) as diagnostic biomarkers in Alzheimer's disease. Clin Chem Lab Med 2011;49:367-374.

-17 Spies PE, Claassen JAHR, Peer PGM, Blankenstein MA, Teunissen CE, Scheltens P, et al: A prediction model to calculate probability of Alzheimer's disease using cerebrospinal fluid biomarkers. Alzheimers Dement 2013; 9:262-268.

18 Skogseth R, Mulugeta E, Ballard C, Rongve A, Nore S, Alves G, et al: Neuropsychiatric correlates of cerebrospinal fluid biomarkers in Alzheimer's disease. Dement Geriatr Cogn Disord 2008;25:559-563.

-19 Kramberger MG, Jelic V, Kåreholt I, Enache D, Eriksdotter Jönhagen M, Winblad B, et al: CSF Alzheimer-markers in depressed elderly with and without Alzheimer's disease. Dement Geriatr Cogn Dis Extra 2012;2:48-56.

20 Engelborghs S, Maertens K, Vloeberghs E, Aerts T, Somers N, Marien P, et al: Neuropsychological and behavioural correlates of CSF biomarkers in dementia. Neurochem Int 2006;48:286-295.

21 Sink KM, Holden KF, Yaffe K: Pharmacological treatment of neuropsychiatric symptoms of dementia: a review of the evidence. JAMA 2005;293:596-608.

-22 Freund-Levi Y, Bloniecki V, Auestad B, Tysen Bäckström A, Lärksäter M, Aarsland D: Galantamine versus risperidone for agitation in people with dementia: a randomized, twelve-week, single-center study. Dement Geriatr Cogn Disord 2014;38:234-244.

23 American Psychiatric Association: Diagnostic and Statistical Manual of Mental Disorders, ed 4. Washington, American Psychiatric Association, 1995.

24 Cummings JL, Mega M, Gray K, Rosenberg-Thompson S, Carusi DA, Gornbein J: The Neuropsychiatric Inventory: comprehensive assessment of psychopathology in dementia. Neurology 1994;44:2308-2314.

-25 Freund-Levi Y, Jedenius E, Tysen-Bäckström AN, Lärksäter M, Wahlund LO, Eriksdotter M: Galantamine versus risperidone treatment of neuropsychiatric symptoms in patients with probable dementia: an open randomised trial. Am J Geriatr Psychiatry 2014;22:341-348.

-26 Folstein MF, Folstein SE, McHugh PR: 'Mini-mental state'. A practical method for grading the cognitive state of patients for the clinician. J Psychiatr Res 1975;12:189-198.

27 Cohen-Mansfield J, Billig N: Agitated behaviors in the elderly. I - A Conceptual review. J Am Geriatr Soc 1986; 34:711-721.

28 Blennow K, Wallin A, Agren H, Spenger C, Siegfried J, Vanmechelen E: Tau protein in cerebrospinal fluid: a biochemical diagnostic marker for axonal degeneration in Alzheimer's disease? Mol Chem Neuropathology 1995;26:231-245.

-29 Vanmechelen E, Vanderstichele H, Davidsson P, Van Kerschaver E, Van Der Perre B, Sjögren M, et al: Quantification of tau phosphorylated at threonine 181 in human cerebrospinal fluid: a sandwich ELISA with a synthetic phosphopeptide for standardization. Neurosci Lett 2000;285:49-52.

-30 Andreasen N, Hesse C, Davidsson P, Minthon L, Wallin A, Winblad B, et al: Cerebrospinal fluid B-amyloid ${ }_{(1-42)}$ in Alzheimer's disease: differences between early- and late-onset Alzheimer disease and stability during the course of disease. Arch Neurol 1999;56:673-680.

-31 Blennow K, Zetterberg H: Cerebrospinal fluid biomarkers for Alzheimer's disease. J Alzheimers Dis 2009;18: 413-417.

32 Senanarong V, Cummings JL, Fairbanks L, Mega M, Masterman DM, O'Connor SM, Strickland TL: Agitation in Alzheimer's disease is a manifestation of frontal lobe dysfunction. Dement Geriatr Cogn Disord 2004;17: 14-20.

-33 Roe CM, Fagan AM, Grant EA, Holtzman DM, Morris JC: CSF biomarkers of Alzheimer disease: 'noncognitive' outcomes. Neurology 2013;81:2028-2031.

-34 Lawlor B: Managing behavioural and psychological symptoms in dementia. B J Psychiatry 2002;181:463-465. 